\title{
¿Nativos o zombis digitales?
}

\author{
Guillermina Ávila García
}

\section{Resumen}

Mi trabajo docente se distingue por el diseño de una secuencia didáctica que permite guiar el proceso de aprendizaje de los estudiantes en modalidad escolarizada. La tecnología la utilizaba como un complemento para el apoyo de dicho proceso. Sin embargo, el primer semestre del año 2020, debido a la pandemia, tomó un rumbo distinto. Se suspendió la educación presencial del cotidiano académico y la tecnología se volvió un parteaguas en el proceso de enseñanza-aprendizaje para las materias que imparto: física y matemáticas.

Palabras clave: aprendizaje, enseñanza, nativos digitales, tecnología.

\section{Digital or ZOMBIE NATIVE?}

\begin{abstract}
My teaching work is distinguished by the design of a didactic sequence that allows me to guide the student's' learning process in face-to-face education. The technology was used as a complement to support this process. However, the first half of 2020, due to the pandemic, took a different course. Face-to-face education was suspended, and technology became a watershed in the teaching-learning process of the subjects I teach: physics and mathematics.
\end{abstract}

Keywords: learning, teaching, digital natives, technology.

Recepción: 30/10/2020. Aprobación: 1/12/2020. Dol: http://doi.org/10.22201/cuaieed.16076079e.2021.22.1.18 
"¿Nativos o zombis digitales?"

Guillermina Ávila García

Vol. 22, Núm. 1, enero-febrero 2021

Revista Digital Universitaria

\section{Guillermina Ávila García}

gavilag@ipn.mx

orcid.org/0000-0001-5229-3384

prezi

Estudió la Licenciatura en Física y Matemáticas. Realizó estudios de Maestría en Ciencia y Tecnología en el Centro de Investigaciones Económicas, Administrativas y Sociales del Instituto Politécnico Nacional. Actualmente estudia el Doctorado en Ciencias en Física educativa en el Centro de Investigación en Ciencia Aplicada y Tecnología Avanzada del Instituto Politécnico Nacional (IPN), es Profesora de Física y Matemáticas en CECYт 11 "Wilfrido Massieu" y en la Escuela Superior de Ingeniería Química e Industrias Extractivas, también ha participado como profesora invitada en la Escuela Superior de Comercio y Administración. Cuenta con experiencia docente desde 2004.

Es miembro activo de la Red de Seminarios Repensar (RSR) y de la Red de Innovación e Investigación Educativas (RIIED), ambas del IPN. Miembro de la Sociedad Mexicana de Computación en la Educación (SOMECE). Ha participado en congresos nacionales e internacionales, entre los que destaca la Conferencia Interamericana de Educación Matemática (CIAEM, 2019), celebrado en Medellín, Colombia; con el trabajo "Modelación con Tracker para el aprendizaje de movimientos en el plano". Su línea de trabajo versa en la innovación educativa de los aprendizajes y el uso inteligente de la tecnología en el aprendizaje de las ciencias.

$\mathrm{M}$

ucho se ha hablado de los nativos digitales, que de acuerdo con Marc Prensky (2001) se caracterizan por haber nacido en la era habilidad consumada, y sienten atracción por todo lo relacionado con las nuevas tecnologías. Esta pandemia ha puesto de manifiesto, en las asignaturas que imparto a nivel medio superior, que no todos los estudiantes nombrados nativos digitales cumplen con esas características en el sentido del aprendizaje.

He escuchado decir que los profesores no tenemos preparación en cuanto al uso de tecnologías, que los estudiantes nos rebasan. En parte coincido, pero también tenemos actualización y en lo personal procuro tomar cursos en apoyo a la enseñanza. Con anterioridad ya usaba la plataforma Classroom para tareas o algunas actividades relacionadas con videos, por ello no me fue difícil incorporarme al trabajo en línea.

Lo complicado empezó con las clases en videosesiones. Que los estudiantes consideraran que la pandemia terminaría pronto implicó no darles 
la importancia adecuada, querían solución a sus dudas en todo momento, sin darse la oportunidad de investigar de forma autónoma. Entonces, en forma presencial, ¿les resolvemos todo?

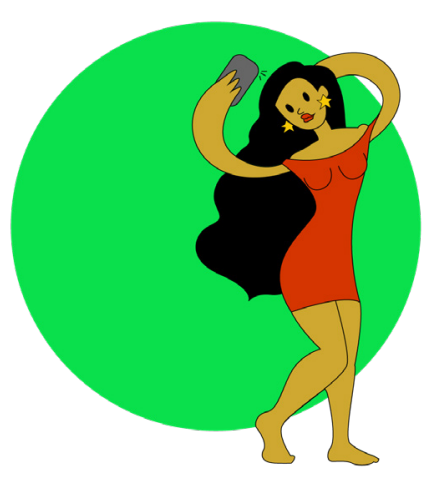

En mensajes de Twitter de diferentes instituciones educativas leía que los estudiantes estaban cansados de tanto trabajo en línea. Entiendo que todas las materias exigen tareas propias, pero ¿acaso no se dejaban en forma presencial? Las clases continuaron en línea, es verdad que faltaba la presencia, pero estamos todos en la misma situación.

Considero que, si tenemos tecnología a nuestro alcance, deber ser usada para investigar y aclarar dudas. Los profesores debemos estar pendientes del aprendizaje, sin embargo, surgieron constantemente cuestiones sencillas como descargar algún software, hacer un video, o subir un archivo en Classroom, con el fin de escribir las observaciones de modo puntual en los posibles errores, para que puedan corregirlas.

Es un reto trabajar en línea, sobre todo cuando los estudiantes no siguen indicaciones, no revisan los comentarios u observaciones, no les gusta explorar, pareciera que siempre están en letargo. Yo considero que no por llamarlos nativos digitales procuran usar lo digital para su aprendizaje.

Desde mi punto de vista, pienso que es importante capacitar a los estudiantes en el uso y manejo de plataformas educativas, con el fin de no distraer la atención de la materia y así mejorar el aprendizaje.

\section{Cómo citar este artículo}

* Ávila García, Guillermina. (2021, enero-febrero). ¿Nativos o zombis digitales? Revista Digital Universitaria (RDU), 22(1). Dol: http://doi.org/10.22201/ cuaieed.16076079e.2021.22.1.18 